\title{
Increased oxygen free radical production during pregnancy may impair vascular reactivity in preeclamptic women
}

\author{
Kazushi Watanabe, Toshitaka Mori, Ai Iwasaki, Chiharu Kimura, Hiroshi Matsushita, Koichi Shinohara \\ and Akihiko Wakatsuki
}

To determine whether enhanced oxidative stress during pregnancy impairs vascular endothelial function and improves after delivery in preeclamptic women, we measured serum parameters of oxidative stress and endothelial function during pregnancy and 1 month after delivery in women with or without preeclampsia. We evaluated 18 participants with uncomplicated pregnancies, 11 with mild preeclampsia and 13 with severe preeclampsia. The plasma concentrations of reactive oxygen metabolite derivatives (d-ROMs) were measured, and the biological antioxidant potential (BAP) was determined to evaluate the oxygen free radicals and antioxidants, respectively. Flow-mediated vasodilation (FMD) was also assessed as a marker of endothelial function. FMD was decreased significantly in both preeclamptic groups compared with control during pregnancy. FMD did not change after delivery in the control group, but it significantly increased after delivery in both the mildly and severely preeclamptic groups, nearing control levels 1 month after delivery (mild, $6.5 \pm 3.6-9.0 \pm 3.5 \%$; severe, $4.3 \pm$ $3.3-9.7 \pm 2.6 \%$ ). No changes in d-ROM concentrations were observed in the control group; however, the concentrations in both the mildly and severely preeclamptic groups significantly decreased to normal levels 1 month after delivery (mild, 562.0 \pm 106.5-430.5 \pm 90.5 CARR U (Carratelli units); severe, 681.0 $\pm 239.0-411.8 \pm 69.7$ CARR U). The plasma BAP levels did not change significantly in all three groups. A negative correlation between FMD and d-ROM concentrations was observed in the preeclamptic group, but not in the control group $(r=-0.497 ; \boldsymbol{P}<0.05)$. Our findings indicated that enhanced oxidative stress during pregnancy may impair endothelial function and improve after delivery in preeclamptic women. Hypertension Research (2013) 36, 356-360; doi:10.1038/hr.2012.208; published online 17 January 2013

Keywords: Preeclampsia; oxidative stress; antioxidant; endothelial dysfunction

\section{INTRODUCTION}

Preeclampsia, a complex generalized syndrome in pregnancy characterized by hypertension and proteinuria, remains a major cause of maternal and fetal morbidity and mortality. ${ }^{1}$ Endothelial dysfunction is considered a basic pathological event that occurs with preeclampsia. ${ }^{2}$ This endothelial damage is mediated in part by oxidative stress that results from increased oxygen free radicals and/or decreased antioxidant levels. ${ }^{3}$ Endothelial injury reduces vasodilator (that is, prostacyclin and nitric oxide $(\mathrm{NO})$ ) production and increases vasoconstrictor (that is, endothelin-1 and thromboxane) production, thereby resulting in vasospasms.

The high maternal and fetal oxygen demand during pregnancy increases oxidative metabolism, which promotes free radical generation. ${ }^{4}$ Superoxide anions produced by the placental mitochondria appear to be an important source of oxidative stress, which contributes to an overall increase in maternal blood and placental lipid peroxidation in preeclamptic women. ${ }^{5-8}$ We previously suggested that increased oxygen free radicals may originate from the placenta in preeclamptic women as these free radicals decrease after delivery. ${ }^{9}$

The measurement of flow-mediated vasodilation (FMD) of conduit arteries is a widely used test of endothelial function. ${ }^{10,11}$ Endothelial dysfunction is an early event in atherogenesis and exists in patients with coronary artery disease and other cardiovascular risk factors. ${ }^{10,12}$ An increase in vascular shear stress, which results from an increase in blood flow and the concomitant release of NO, has been identified as a mechanism for FMD. ${ }^{13,14}$ We previously found that FMD of the brachial artery, a known marker of vascular endothelial function, is decreased in preeclamptic women. ${ }^{15,16}$

Pre-existing chronic hypertension, obesity, diabetes mellitus and systemic lupus erythematosus may initiate vasospasms secondary to endothelial dysfunction, and endothelial dysfunction does not completely improve after delivery. Pregnancies in patients with these pre-existing conditions have a greater risk of preeclampsia, which frequently becomes apparent during pregnancy. However, it is unclear whether enhanced oxidative stress during pregnancy impairs vascular 
endothelial function and whether it improves after delivery in preeclamptic women without these pre-existing conditions. To address this, we measured serum parameters of oxidative stress and endothelial function during pregnancy and 1 month after delivery in women with or without preeclampsia.

\section{METHODS}

\section{Study participants}

Between 1 April 2008 and 31 March 2010, we recruited 11 participants with mild preeclampsia, 13 participants with severe preeclampsia and 18 normal participants with uncomplicated pregnancies. The selection criteria for participants with mild preeclampsia included a systolic blood pressure (BP) $\geqslant 140$ and $<160 \mathrm{~mm} \mathrm{Hg}$ or a diastolic BP $\geqslant 90$ and $<110 \mathrm{~mm} \mathrm{Hg}$ after 20 weeks of gestation and proteinuria ( $\geqslant 300 \mathrm{mg}$ and $<2 \mathrm{~g}$ protein per $24 \mathrm{~h}$ ). The selection criteria for participants with severe preeclampsia included a systolic $\mathrm{BP} \geqslant 160 \mathrm{~mm} \mathrm{Hg}$ or diastolic $\mathrm{BP} \geqslant 110 \mathrm{~mm} \mathrm{Hg}$ after 20 weeks of gestation and proteinuria ( $\geqslant 2 \mathrm{~g}$ protein per $24 \mathrm{~h}$ ). None of the preeclamptic participants showed signs of hemolysis, elevated liver enzymes, and low platelet syndrome. Participants with uncomplicated pregnancies, matched for maternal and gestational age, were used as controls. Each control group participant had a normal pregnancy course and full-term delivery. None of the participants smoked, used caffeine or alcohol, or had a history of thyroid disease, liver disease, diabetes mellitus, hypertension, hyperlipidemia, or were taking any medication known to influence lipoprotein metabolism. In the present study, all participants delivered by cesarean section before the onset of labor. All control group participants underwent cesarean section due to a previous cesarean section or breech presentation. Written informed consent was obtained from each participant. This study was approved by the Ethics Committee of Aichi Medical University School of Medicine.

\section{Derivatives of reactive oxygen metabolites and biological antioxidant potential (BAP)}

Blood samples were collected from each participant between 0900 hours and 1100 hours after a 12-h fasting period before delivery in the third quarter and 1 month after delivery. Serum derivatives of reactive oxygen metabolites (d-ROMs), a marker of oxidant status, and the serum BAP were analyzed using a Free Radical Analytical System 4 ((Diacron, Grosseto, Italy). ${ }^{17-20}$ Organic hydroperoxide serum measurements were taken to determine the levels of free radicals and the source. Under acidic conditions, hydroperoxides react with iron, a transition metal that is liberated from proteins, and they are converted into alkoxyl and peroxyl radicals. These radicals can oxidize an additive to its corresponding radical cation species. The concentrations of these persistent species were measured by spectrophotometry $(505 \mathrm{~nm})$. The results for all d-ROM measurements were expressed in Carratelli units (CARR U).

The BAP serum levels were also measured to determine the antioxidant levels. The BAP test is based on the ability of a colored solution containing a source of ferric ions bound to a special chromogenic substrate to decolorize when ferric ions are reduced to ferrous ions, which occurs when a reducing/ antioxidant source is added. The concentration is then measured by spectrophotometry $(505 \mathrm{~nm})$. In the present study, an imbalance between increased oxygen free radicals and the antioxidative processes was determined and used to calculate d-ROMs and BAP concentrations in both maternal and postpartum blood.

\section{Endothelial function}

All participants were required to rest in a supine position for $10 \mathrm{~min}$ before the study. A high-resolution, Doppler ultrasonography machine (VolusonE8, GE Healthcare, Nagoya City, Japan) with a 10-MHz transducer was used to image the right brachial artery and measure the vasodilatory responses. A nontortuous segment of the brachial artery was scanned longitudinally $4-5 \mathrm{~cm}$ above the elbow, as this was the region where the clearest image could be obtained. Once the adequate transducer position was determined, the skin was marked and the arm was kept in a constant position for the remainder of the study. After baseline images of the brachial artery were obtained and the arterial flow velocity was determined, a BP cuff encircling the proximal portion of the arm was inflated to $250 \mathrm{~mm} \mathrm{Hg}$ for $5 \mathrm{~min}$ and then suddenly deflated. Increased blood flow following sudden cuff deflation (that is, reactive hyperemia) results in flow-mediated vasodilatation (FMD). ${ }^{21}$ The arterial flow velocity was determined again and, $1 \mathrm{~min}$ after cuff deflation, the brachial artery was imaged. BP and heart rate were monitored and recorded throughout the study. The diameter of the brachial artery was measured from the anterior to the posterior interface between the media and adventitia (' $m$ line') at end diastole, incident with the $\mathrm{R}$ wave on a continuously recorded electrocardiogram. The diameters for four cardiac cycles were determined from the images and the measurements were averaged. All scans were recorded for further analysis. The vessel diameters were measured without prior knowledge of the participant information. FMD was calculated as the percent increase in arterial diameter during hyperemia and used as an index of endothelium-dependent vasodilation. ${ }^{22}$ Intraobserver and interobserver variability for repeat measurements were $0.03 \pm 0.02$ and $0.05 \pm 0.03 \mathrm{~mm}$, respectively, and the variability for FMDs measured on two separate days was $2.1 \pm 0.9 \%$.

\section{Statistical analyses}

SPSS Statistical Software, version 14.0 for Windows (SPSS Inc., Chicago, IL, USA), was used for all statistical analyses. The data are expressed as the mean \pm s.d. The patient characteristics, serum d-ROM and BAP concentrations and FMD were compared using a one-way analysis of variance, followed by Scheffe's test. The patient characteristics, serum d-ROM and BAP concentrations and FMD before and post delivery were compared using the Student's paired $t$-test. $P<0.05$ was considered statistically significant.

\section{RESULTS}

Maternal and gestational age at the timeof the study were similar between both preeclamptic groups (that is, mildly and severely) and the control group. The body mass index was significantly higher in the mildly preeclamptic group compared with the severely preeclamptic and control groups. In addition, plasma uric acid levels were significantly elevated during pregnancy in both preeclamptic groups, but they were higher in the severely preeclamptic group. Hematocrit, aspartate aminotransferase and plasma creatine levels during pregnancy were significantly elevated in the severely preeclamptic group but not in the mild preeclamptic group. In addition, the platelet counts during pregnancy were significantly elevated in the mildly preeclamptic group but not in the severely preeclamptic group. Hematocrit and platelet counts, liver enzyme, blood urea nitrogen and creatine plasma levels measured 1 month after delivery were similar for all three groups (Table 1).

No significant changes in d-ROM plasma concentrations before and 1 month after delivery were observed in the control group. However, those concentrations were significantly lower in both preeclamptic groups, reaching control levels 1 month after delivery (mild, 562.0 $\pm 106.5-430.5 \pm 90.5$ CARR U; severe, 681.0 $\pm 239.0-$ 411.8 \pm 69.7 CARR U; Figure 1). The BAP concentrations before and 1 month after delivery were similar in all three groups (Figure 1).

The systolic BP measured 1 month after delivery was significantly higher in both preeclamptic groups. In contrast, the diastolic BP improved to normal levels in both preeclamptic groups. Finally, no significant difference was found in the brachial artery diameter and blood flow velocity during pregnancy and 1 month after delivery in all three groups. The percent increase in blood flow velocity promoted by reactive hyperemia did not change significantly (Table 2). FMD decreased significantly in both preeclamptic groups compared with the control during pregnancy. FMD in the control group remained the same after delivery, but it increased significantly to control levels 1 month after delivery in both preeclamptic groups (mild, $6.5 \pm 3.6-$ $9.0 \pm 3.5 \%$; severe, $4.3 \pm 3.3-9.7 \pm 2.6 \%$; Figure 2 ). A negative correlation between FMD and d-ROM concentrations was observed in the 


\begin{tabular}{|c|c|c|c|c|c|c|}
\hline & \multicolumn{2}{|c|}{ Normal $(\mathrm{n}=18)$} & \multicolumn{2}{|c|}{ Mild preeclampsia $(\mathrm{n}=11)$} & \multicolumn{2}{|c|}{ Severe preeclampsia $(n=13)$} \\
\hline & $\begin{array}{c}\text { During } \\
\text { pregnancy }\end{array}$ & $\begin{array}{c}1 \text { month after } \\
\text { delivery }\end{array}$ & $\begin{array}{l}\text { During } \\
\text { pregnancy }\end{array}$ & $\begin{array}{c}1 \text { month after } \\
\text { delivery }\end{array}$ & $\begin{array}{c}\text { During } \\
\text { pregnancy }\end{array}$ & $\begin{array}{c}1 \text { month after } \\
\text { delivery }\end{array}$ \\
\hline Age (years) & $33.5 \pm 4.6$ & - & $31.3 \pm 5.4$ & - & $31.5 \pm 5.2$ & - \\
\hline Gestational age at test (weeks) & $34.6 \pm 5.0$ & - & $34.7 \pm 3.3$ & - & $33.3 \pm 2.9$ & - \\
\hline BMI $\left(\mathrm{kgm}^{-2}\right)$ & $19.7 \pm 2.2$ & - & $23.5 \pm 7.6^{a}$ & - & $22.2 \pm 3.9$ & - \\
\hline Platelet $\left(\times 10^{4}\right)$ & $24.3 \pm 5.5$ & $28.1 \pm 10.8$ & $31.1 \pm 9.2^{\mathrm{a}, \mathrm{e}}$ & $31.4 \pm 12.8$ & $24.9 \pm 8.2$ & $37.9 \pm 17.5^{9}$ \\
\hline AST $\left(|U|^{-1}\right)$ & $16.7 \pm 4.0$ & $21.4 \pm 6.9$ & $13.8 \pm 3.7$ & $18.5 \pm 6.2$ & $20.0 \pm 4.4^{\mathrm{a}, \mathrm{d}}$ & $20.7 \pm 6.6$ \\
\hline ALT $\left(I \cup I^{-1}\right)$ & $10.6 \pm 5.0$ & $16.2 \pm 2.2^{f}$ & $8.2 \pm 3.2$ & $21.0 \pm 11.8^{\mathrm{g}}$ & $12.7 \pm 9.0$ & $21.2 \pm 10.3 \mathrm{~g}$ \\
\hline BUN (mgdl-1) & $7.8 \pm 2.4$ & $11.5 \pm 3.3$ & $7.4 \pm 2.1$ & $10.9 \pm 3.3^{g}$ & $12.5 \pm 2.9^{b, d}$ & $10.3 \pm 2.4^{4}$ \\
\hline Creatinine $\left(\mathrm{mg} \mathrm{dl}^{-1}\right)$ & $0.44 \pm 0.06$ & $0.53 \pm 0.07$ & $0.50 \pm 0.10$ & $0.57 \pm 0.08^{f}$ & $0.62 \pm 0.11^{\mathrm{b}, \mathrm{d}}$ & $0.58 \pm 0.08^{f}$ \\
\hline Uric acid (mg dl-1) & $4.0 \pm 0.8$ & $4.4 \pm 0.6$ & $4.9 \pm 1.1^{\mathrm{a}}$ & $5.6 \pm 1.2^{\mathrm{a}, \mathrm{f}}$ & $6.5 \pm 1.4^{\mathrm{b}, \mathrm{d}}$ & $5.3 \pm 0.99$ \\
\hline
\end{tabular}

Abbreviations: ALT, alanine aminotransferase; AST, asparate aminotransferase; BMI, body mass index; BP, blood pressure; BUN, blood urea nitrogen.

Data are expressed as mean \pm s.d.

a $P<0.05$, b $P<0.01$ as compared with normal

${ }^{\mathrm{c}} P<0.05, \mathrm{~d} P<0.01$ as compared with mild preeclampsia.

${ }^{\mathrm{e}} P<0.05$ as compared with severe preeclampsia.

${ }_{\mathrm{f}} P<0.05, \mathrm{~g} P<0.01$ as compared with during pregnancy.
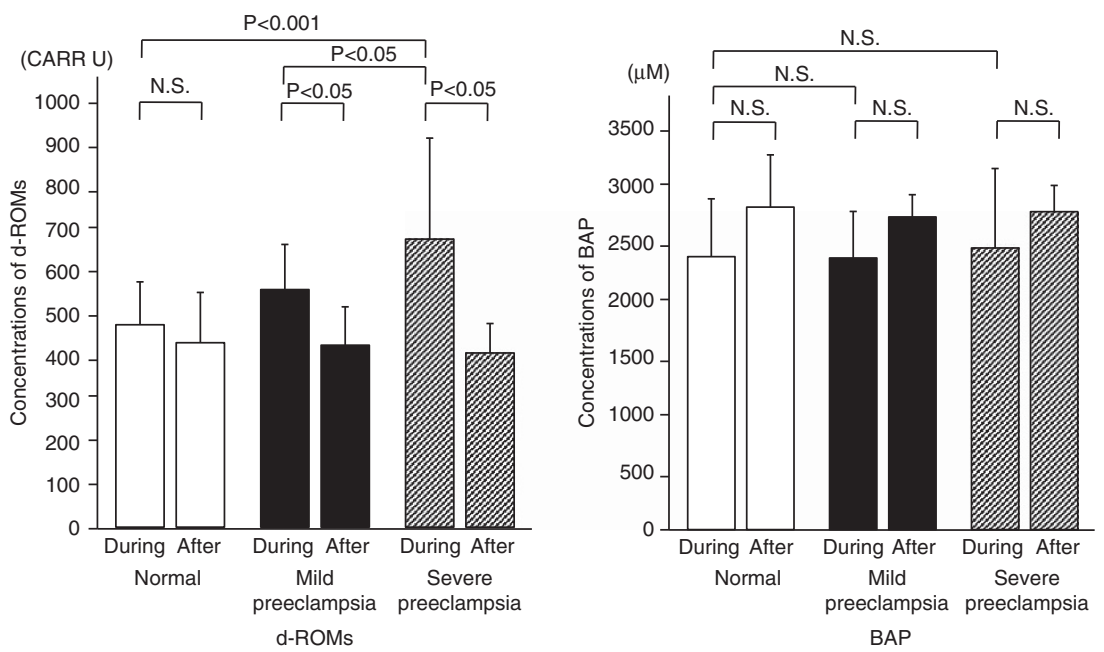

BAP

Figure 1 Concentrations of derivatives of reactive oxygen metabolites (d-ROMs) and biological antioxidant potential (BAP) in maternal blood during pregnancy and 1 month after delivery in the control group and both preeclamptic women. Open, closed and diagonally hatched columns represent healthy women with uncomplicated pregnancies, mild preeclamptic women and severe preeclamptic women, respectively. During and after indicate during pregnancy and 1 month after delivery, respectively. N.S. indicates not significant. Data are expressed as mean \pm s.d.

Table 2 Blood pressure, heart rate, artery diameter and blood flow

\begin{tabular}{|c|c|c|c|c|c|c|}
\hline & \multicolumn{2}{|c|}{ Normal } & \multicolumn{2}{|c|}{ Mild preeclampsia } & \multicolumn{2}{|c|}{ Severe preeclampsia } \\
\hline & During pregnancy & 1 month after delivery & During pregnancy & 1 month after delivery & During pregnancy & 1 month after delivery \\
\hline Systolic BP (mm Hg) & $111.9 \pm 14.0$ & $114.4 \pm 8.7$ & $141.9 \pm 11.6^{\mathrm{b}}$ & $131.7 \pm 10.3^{b}$ & $164.8 \pm 20.8^{\mathrm{b}, \mathrm{c}}$ & $126.1 \pm 9.8^{\mathrm{a}, \mathrm{d}}$ \\
\hline Diastolic BP (mm Hg) & $75.0 \pm 6.9$ & $73.4 \pm 8.8$ & $84.9 \pm 9.7^{a}$ & $80.2 \pm 7.1$ & $103.8 \pm 8.7^{\mathrm{b}, \mathrm{c}}$ & $80.2 \pm 8.1^{\mathrm{e}}$ \\
\hline Heart rate (beats per minute) & $72.7 \pm 3.9$ & $72.6 \pm 6.3$ & $73.0 \pm 2.8$ & $73.2 \pm 10.7$ & $78.1 \pm 7.5$ & $77.9 \pm 6.6$ \\
\hline Baseline diameter (mm) & $3.4 \pm 0.3$ & $3.1 \pm 0.3$ & $3.6 \pm 0.3$ & $3.1 \pm 0.2$ & $3.7 \pm 0.1$ & $3.2 \pm 0.2^{\mathrm{e}}$ \\
\hline Baseline flow $\left(\mathrm{ml} \mathrm{min}^{-1}\right)$ & $183 \pm 89$ & $116 \pm 45^{d}$ & $209 \pm 79$ & $115 \pm 47^{d}$ & $218 \pm 87$ & $149 \pm 53^{d}$ \\
\hline Hyperemic flow ( $\left.\mathrm{ml} \mathrm{min}^{-1}\right)$ & $346 \pm 98$ & $312 \pm 149$ & $417 \pm 108$ & $317 \pm 96$ & $368 \pm 117$ & $408 \pm 219$ \\
\hline
\end{tabular}

Abbreviation: BP, blood pressure.

Data are expressed as mean $\pm s . d$

a $P<0.05,{ }^{b} P<0.01$ as compared with normal,

${ }^{c} P<0.01$ as compared with mild preeclampsia.

$\mathrm{d} P<0.05$, ${ }^{\mathrm{e}} P<0.01$ as compared with during pregnancy. 


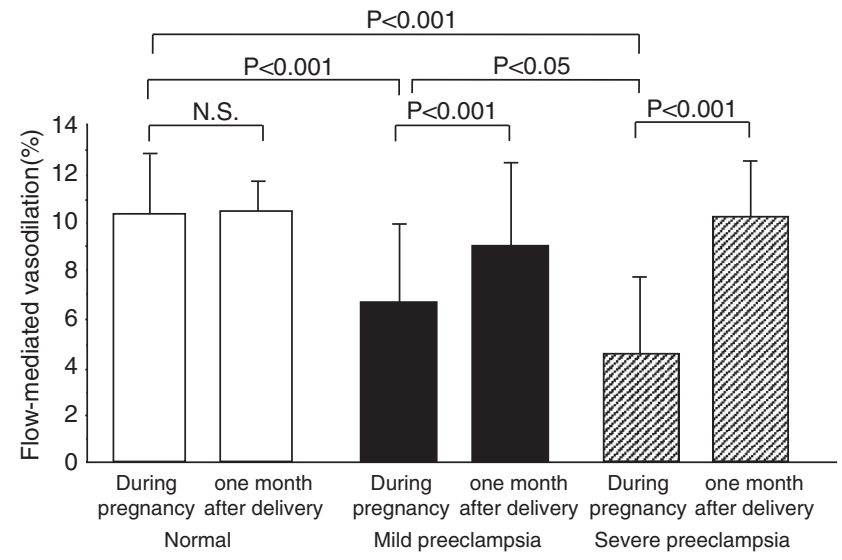

Figure 2 Flow-mediated vasodilation during pregnancy and 1 month after delivery in the control group and both preeclamptic women. Open, closed and diagonally hatched columns represent healthy women with uncomplicated pregnancies, mild preeclamptic women, and severe preeclamptic women, respectively. N.S. indicates not significant. Data are expressed as mean \pm s.d.

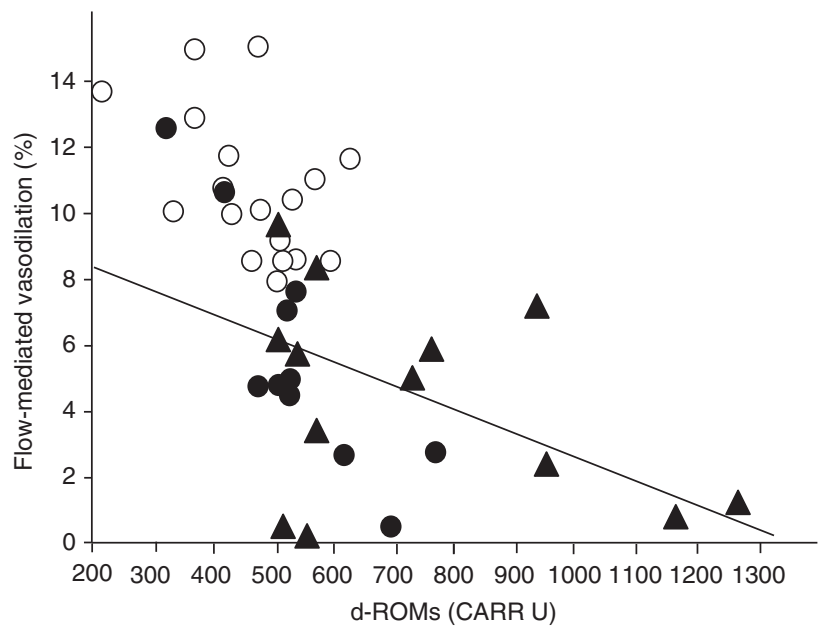

Figure 3 Relationship between concentrations of derivatives of reactive oxygen metabolites (d-ROMs) and flow-mediated vasodilation during pregnancy in the control group and preeclamptic women. Open circles, closed circles and closed triangles represent healthy women with uncomplicated pregnancies, mild preeclamptic women and severe preeclamptic women, respectively. $r=-0.497 ; P<0.05$.

preeclamptic group, but not in the control group $(r=-0.497$; $P<0.05$ ) (Figure 3).

\section{DISCUSSION}

Oxygen free radical production is commonly increased in women with preeclampsia. Although a physiological balance between lipid peroxides and antioxidative processes is maintained during a normal pregnancy, ${ }^{23}$ an imbalance may occur, thereby increasing the oxidative stress in preeclamptic women. In the present study, we found that d-ROM concentrations in the blood were significantly higher in the severely preeclamptic group, but not in the mildly preeclamptic group, compared with the control group. In contrast, BAP concentrations did not change significantly across all three groups. These results indicate that the balance between oxygen free radicals and antioxidative processes is maintained in mildly preeclamptic women, but not in severely preeclamptic women. The results also indicate that this imbalance is dependent on increased oxygen free radical production and not on decreased antioxidative processes. Furthermore, we investigated whether enhanced oxidative stress improved after delivery. We demonstrated that plasma d-ROM levels 1 month after delivery in the severe preeclamptic group normalized to control levels. These results indicate that oxidative stress is greater during pregnancy but that it normalizes after delivery in severely preeclamptic women. Although the d-ROM concentrations in the mildly preeclamptic and control groups were not significantly different during pregnancy, the levels in the mildly preeclamptic group were reduced 1 month after delivery. These results indicate that oxidative stress is slightly higher during pregnancy in mildly preeclamptic women. We believe that the severity of preeclampsia during pregnancy increases with oxidative stress.

$\mathrm{NO}$ is an endothelium-derived relaxing factor that is released in response to increased blood flow during reactive hyperemia. Because several NO synthase inhibitors inhibit endothelium-dependent vasodilation, FMD appears to represent a vasodilation-dependent effect mediated by endothelium-derived NO. ${ }^{14}$ In the present study, a negative correlation between FMD and d-ROM concentrations was observed in the preeclamptic group. We also found that d-ROMs and FMD in both preeclamptic groups normalized 1 month after delivery, suggesting that oxidative stress during pregnancy may impair endothelial function. In contrast, Hamad et al. ${ }^{24}$ reported that FMD and endothelium-independent glycerin trinitrate-induced dilatation, a marker of vascular muscle cell function, are decreased even 1 year after a preeclamptic pregnancy. These results indicate that endothelial dysfunction remains after delivery, or that after improving post delivery, it decreases again soon thereafter. In their study, endothelial dysfunction that remains after delivery is at an advanced stage due to decreased glycerin trinitrate-induced dilatation, a marker of vascular muscle cell function. Indeed, FMD was lower in their study than in ours. The authors themselves note that the possibility of generalized impairment in vascular muscle cell responsiveness contributing to the observed low FMD cannot be excluded. In our view, the endothelial and vascular muscle cell dysfunction may have preexisted before pregnancy as chronic hypertension in their study. We speculate that if conditions such as obesity, dysregulation of adipocytokines, and oxidative stress persist after delivery, they may impair endothelial function in the future. Indeed, the body mass index was significantly higher in the preeclamptic group compared with the control group 1 year after a preeclamptic pregnancy in their study. ${ }^{24}$ Consistent with this, in our preliminary study, endothelial dysfunction failed to improve, and it remained after delivery in pregnant women with pre-existing conditions such as chronic hypertension.

Yinon et al. ${ }^{25}$ reported that FMD was significantly reduced in women with a history of early-onset preeclampsia and normotensive intrauterine fetal growth restriction, but not in women with a history of late-onset preeclampsia. In a previous study, we defined preeclampsia with an onset before the 32nd week of gestation as early-onset preeclampsia, and preeclampsia with an onset after the 32nd week was defined as late-onset preeclampsia. We found that FMD in patients with early-onset preeclampsia was significantly lower than in those with late-onset preeclampsia. ${ }^{16}$ This indicates that endothelial dysfunction is more severe in early-onset preeclampsia than in late-onset preeclampsia. Given that FMD was not tracked long term after delivery in the present study, further studies will be needed to address this possibility. 


\section{CONFLICT OF INTEREST}

The authors declare no conflict of interest.

1 Mackay AP, Berg CJ, Atrash HK. Pregnancy-related mortality from preeclampsia and eclampsia. Obstet Gynecol 2001; 97: 533-538.

2 Roberts JM, Taylor RN, Musci TJ. Preeclampsia: an endothelial cell disorder. Am J Obstet Gynecol 1989; 161: 1200-1204.

3 Hubel CA, Roberts JM, Taylor NR, Musci TJ, Rogers GM, Mclaughlin MK. Lipid peroxidation in pregnancy: new perspectives on preeclampsia. Am J Obstet Gynecol 1989; 161: 1025-1034.

4 Wisdom SJ, Wilson R, Mckillop JH, Walker JJ. Antioxidant system in normal pregnancy and pregnancy induced hypertension. Am J Obstet Gynecol 1991; 165 1701-1704.

5 Wang Y, Walsh SW, Kay HH. Placental lipid peroxides and thromboxane are increased and prostacyclin is decreased in women with preeclampsia. Am J Obstet Gynecol 1992; 167: 946-949.

6 Poranen AK, Ekblad U, Uotila P, Ahotupa M. Lipid peroxidation and antioxidants in normal and pre-eclamptic pregnancies. Placenta 1996; 17: 401-405.

7 Wang Y, Walsh SW. Placental mitochondria as a source of oxidative stress in preeclampsia. Placenta 1998; 19: 581-586.

8 Gurjit K, Soumya M, Alka S, Rajendra P. Alterations in lipid peroxidation and antioxidant status in pregnancy with preeclampsia. Mol Cell Biochem 2008; 313: 37-44.

9 Fujimaki A, Watanabe K, Mori T, Kimura C, Shinohara K, Wakatsuki A. Placental oxidative DNA damage and its repair in preeclamptic women with fetal growth restriction. Placenta 2011; 32: 367-372.

10 Celermajer DS, Sorensen KE, Gooch VM, Spiegelhalter DJ, Miller OI, Sullivan ID, Lloyd JK, Deanfield JE. Non-invasive detection of endothelial dysfunction in children and adults at risk of atherosclerosis. Lancet 1992; 340: 1111-1115.

11 Takase B, Uehata A, Akima T, Nagai T, Nishioka T, Hamabe A, Satomura K, Ohsuzu F, Kurita A. Endothelium-dependent flow-mediated vasodilation in coronary and branchia arteries in suspected coronary artery disease. Am J Cardiol 1998; 82: 1535-1539.

12 Vogel RA. Measurement of endothelial function by branchial artery flow-mediated vasodilation. Am J Cardiol 2001; 88: 31E-34E.
13 Doshi SN, Naka KK, Payne N, Jones CJ, Ashton M, Lewis MJ, Doodfellow J. Flow-mediated vasodilation following wrist and upper arm occlusion in humans: the contribution of nitric oxide. Clin Sci 2001; 101: 629-635.

14 Joannides R, Haefeli WE, Linder L, Richard V, Bakkali E, Thuillez C, Luscher TF. Nitric oxide is responsible for flow-dependent dilation of human peripheral conduit arteries in vivo. Circulation 1995; 91: 1314-1319.

15 Mori T, Shinohara K, Wakatsuki A, Watanabe K, Fujimaki A. Adipocytokines and endothelial function in preeclamptic women. Hypertension Res 2010; 33: 250-254.

16 Watanabe K, Mori T, Iwasaki A, Kimura C, Matsushita H, Shinohara K, Wakatsuki A. Increased oxidant generation in the metabolism of hypoxanthine to uric acid and endothelial dysfunction in early-onset and late-onset preeclamptic women. J MaternFetal Neonatal Med 2012; 25: 2662-2666.

17 Trotti R, Carratelli M, Barbieri M. Performance and clinical application of a new, fast method for the detection of hydroperoxides in serum. Panminerva Med 2002; 44: 37-40.

18 Parmigiani S, Payer C, Massari A, Bussolati G, Bevilacqua G. Normal values of reactive oxygen metabolites on the cord-blood of fullterm infants with a colorimetric method. Acta Biomed Ateneo Parmense 2000; 71: 59-64.

19 Cesarone MR, Belcaro G, Carratelli M, Cornelli U, De Sanctis MT, Incandela I, Barsott A, Terranova R, Nicolaides A. A simple test to monitor oxidative stress. Int Angio 1998; 18: 127-130.

20 Vallance P, Leone A, Calver A, Collier J, Moncada S. Accumulation of an endogenous inhibitor of NO synthesis in chronic renal failure. Lancet 1992; 339: 572-575.

21 Rubanyi GM, Romero JC, Vanhoutte PM. Flow-induced release of endothelium-derived relaxing factor. Am J Physiol 1986; 250: H1145-H1149.

22 Stein JH, Keevil JG, Wiebe DA, Aeschlimann S, Folts JD. Purple grape juice improves endothelial function and reduces the susceptibility of LDL cholesterol to oxidation in patients with coronary artery disease. Circulation 1999; 100: 1050-1055.

23 Wang Y, Walsh SW, Gu J, Zang J. The imbalance between thromboxane and prostacyclin in preeclampsia is associated with an inbalance between lipid peroxides and vitamin E in maternal blood. Am J Obstet Gynecol 1991; 165: 1695-1700.

24 Hamad RR, Eriksson MJ, Silveira A, Hamsten A, Bremme K. Decreased flow-mediated dilation is present 1 year after a pre-eclamptic pregnancy. J Hypertens 2007; 25 2301-2307.

25 Yinon Y, Kingdom J, Odutayo A, Moineddin R, Drewlo S, Lai V, Cherney D, Hladunewich M. Vascular dysfunction in women with a histoly of preeclampsia and intrauterine growth restriction insights into future vascular risk. Circulation 2010; 122 1846-1853. 